\title{
A NOTE ON PSEUDOVARIETIES OF COMPLETELY REGULAR SEMIGROUPS
}

\author{
JORGE ALMEIDA AND ALFREDO COSTA
}

\begin{abstract}
A paper of Almeida and Trotter on completely regular semigroups makes essential use of free profinite semigroupoids over profinite graphs with infinitely many vertices. It has since been shown that such structures must be handled with great care. In this note, it is verified that the required properties hold for the profinite graphs considered by Almeida and Trotter, and thus the gap is filled.
\end{abstract}

\section{INTRODUCTION}

In [4] one finds a systematic study of relatively free profinite categories and relatively free profinite semigroupoids generated by profinite graphs with a finite number of vertices. The case of profinite graphs with an infinite number of vertices is more delicate, as highlighted in [1. The main problem is that in the infinite-vertex case, the topological closure of the subsemigroupoid generated by the graph may not be a subsemigroupoid of the corresponding free profinite semigroupoid. This problem was overlooked in [3] and [2, where infinite-vertex free profinite semigroupoids are seriously considered for the first time as a tool to be used in the study of relatively free profinite semigroups. While their role in [3] can be considered marginal, in [2] some specific relatively free profinite semigroupoids with an infinite number of vertices play a key role in the proofs of the main results remain valid by establishing that, for the infinite-vertex graphs which are used in the proofs, the semigroupoids generated by the graphs are dense in the corresponding relatively free profinite semigroupoids.

\section{The free profinite semigroupoid Generated By $\partial_{X} P$}

For general background on pseudovarieties of semigroups and of semigroupoids, and on their relatively free profinite structures, see 6 . We also adopt the notation of 2 , which we recall here for the reader's benefit. Let $A$ be a finite alphabet and let $\mathrm{V}$ be a pseudovariety of semigroups. The free pro- $\mathrm{V}$ semigroup generated by $A$ is denoted by $\bar{\Omega}_{A} \mathrm{~V}$, and the subsemigroup of $\bar{\Omega}_{A} \mathrm{~V}$ generated by $A$ is denoted by $\Omega_{A} \mathrm{~V}$. Suppose that $\mathrm{V}$ contains the pseudovariety $\mathrm{SI}$ of finite semilattices. This hypothesis enables us to consider the content, which is a continuous homomorphism $c$ from $\bar{\Omega}_{A} \mathrm{~V}$

1991 Mathematics Subject Classification. primary 20M07; secondary 20M17, 20M05, 22A15.

Key words and phrases. Completely regular semigroups, pseudovarieties, free profinite semigroups, free profinite semigroupoids, profinite graphs.

This research was partially supported by Centro de Matemática da Universidade do Porto (CMUP) [J. Almeida] and Centro de Matemática da Universidade de Coimbra (CMUC) [A. Costa], both funded by the European Regional Development Fund, through the programme COMPETE, and by the Portuguese Government through Fundação para a Ciência e a Tecnologia (FCT), under, respectively, the projects PEst-C/MAT/UI0144/2013 and PEst-C/MAT/UI0324/2013. 
to the union-semilattice of subsets of $A$ that maps each letter $a \in A$ to the singleton set $\{a\}$. The content function extends to $\left(\bar{\Omega}_{A} \mathrm{~V}\right)^{1}$ by letting $c(1)=\emptyset$. Suppose also that $\bar{\Omega}_{A} \vee$ has $0, \overline{0}, 1, \overline{1}$ functions, which means that, for every $w \in \bar{\Omega}_{A} \mathrm{~V}$, there are factorizations $w=w_{0} a w^{\prime}=w^{\prime \prime} b w_{1}$ in $\left(\bar{\Omega}_{A} \mathrm{~V}\right)^{1}$ such that $a, b \in A$ and

$$
c(w)=c\left(w_{0}\right) \uplus\{a\}=\{b\} \uplus c\left(w_{1}\right),
$$

where the factors $w_{0}, a, b, w_{1}$ are unique. We put $0(w):=w_{0}, 1(w):=w_{1}, \overline{0}(w):=a$, and $\overline{1}(w):=b$. Note that the functions $0, \overline{0}, 1, \overline{1}$ are continuous.

Let $X$ be a subset of $A$ with at least two elements. Consider a subset $P$ of $\bar{\Omega}_{A} \mathrm{~V}$ such that $c(u) \subseteq X$ for every $u \in P$. We define a graph ${ }^{1} \partial_{X} P$ on the set of all factors $v \in\left(\bar{\Omega}_{A} \mathrm{~V}\right)^{1}$ of elements of $P$ such that $|X \backslash c(v)| \in\{1,2\}$. The vertices are those $v$ such that $|X \backslash c(v)|=2$ and the edges are the remaining elements of $\partial_{X} P$. The adjacency functions are $\alpha=0$ and $\omega=1$, where, in a graph, we respectively denote by $\alpha(u)$ and $\omega(u)$ the source and the target of an edge $u$. This definition of $\partial_{X} P$ is taken from [2, Subsection 3.1], where the necessary hypothesis that the content of all elements of $P$ is contained in $X$ is not made explicit, but is implicitly used.

Profinite graphs are defined in [2] as being the inverse limits of finite graphs, in the category of topological graphs, with finite graphs having the discrete topology. It is folklore that the profinite graphs are precisely the topological graphs whose topology is a Boolean space (a proof can be found in [7.) It follows immediately from this characterization that the graph $\partial_{X} P$ is profinite if $P$ is a closed subset of $\bar{\Omega}_{A} \mathrm{~V}$, which happens in particular when $P=\bar{\Omega}_{X} \mathrm{~V}$, the case considered in the main results of $[2]$.

Let $\mathrm{W}$ be a pseudovariety of finite semigroupoids. We adopt the definition from [1] of a pro-W semigroupoid as being a compact semigroupoid which is residually in $\mathrm{W}$ in the sense that, for every pair $u, v$ of distinct elements of $S$, there is a continuous semigroupoid homomorphism $\varphi: S \rightarrow F$ into a semigroupoid $F$ of W satisfying $\varphi(u) \neq \varphi(v)$. For the case where $\mathrm{W}$ is the pseudovariety $\mathrm{Sd}$ of all finite semigroupoids, one uses "profinite" as a synonym of "pro-W". We remark that there is an unpublished example due to G. Bergman (mentioned in [5]) of an infinitevertex semigroupoid which is profinite according to this definition, but which is not an inverse limit of finite semigroupoids.

Let $\Gamma$ be a profinite graph and let $W$ be a pseudovariety of semigroupoids. The free pro-W semigroupoid generated by $\Gamma$, denoted $\bar{\Omega}_{\Gamma} \mathrm{W}$, is a pro-W semigroupoid, together with a continuous graph homomorphism $\iota: \Gamma \rightarrow \bar{\Omega}_{\Gamma} \mathrm{W}$, with the following property: for every continuous graph homomorphism $\varphi: \Gamma \rightarrow F$ into a semigroupoid of $\mathrm{W}$, there is a unique continuous semigroupoid homomorphism $\hat{\varphi}: \bar{\Omega}_{\Gamma} \mathrm{W} \rightarrow F$ such that $\hat{\varphi} \circ \iota=\varphi$. It is easy to show that this semigroupoid is indeed unique, up to isomorphism of compact semigroupoids, and a proof of its existence is made in [1] by a reduction to the finite-vertex case treated in [4. Moreover, if $\mathrm{W}$ contains nontrivial semigroups, we may assume (as we do from hereon) that $\iota$ is the inclusion mapping [1. The subsemigroupoid of $\bar{\Omega}_{\Gamma} \mathrm{W}$ generated by $\iota(\Gamma)$ is denoted by $\Omega_{\Gamma} \mathrm{W}$. If $\Gamma$ has a finite number of vertices then $\Omega_{\Gamma} \mathrm{W}$ is dense in $\bar{\Omega}_{\Gamma} \mathrm{W}$ [4]. However, in general, that property fails, and it is only by iterating transfinitely algebraic and topological closures that one reachs the profinite semigroupoid $\bar{\Omega}_{\Gamma} \mathrm{W}[1]$.

\footnotetext{
${ }^{1}$ Throughout this paper, by graph we mean a directed graph.
} 
As remarked in [4], if $\Gamma$ is a finite graph, then $\bar{\Omega}_{\Gamma} \mathrm{W}$ is metrizable. The following is a generalization of this fact, which will be used later on.

Proposition 2.1. Let $\Gamma$ be a profinite graph and let $\mathrm{W}$ be a pseudovariety of semigroupoids. If $\Gamma$ is metrizable, then so is $\bar{\Omega}_{\Gamma} \mathrm{W}$.

For the proof of Proposition 2.1 one uses the next folklore result, for which we do not have a direct reference. However, a proof is implicit in the proof of 8 , Corollary 1.1.13] (there the reference is made to profinite second countable spaces, but by Urysohn's metrization theorem [9. Theorem 23.1], a profinite space is second countable if and only if it is metrizable.) In the statement we use the notation in 8 , Chapter I] for inverse systems.

Proposition 2.2. Let the topological space $X$ be the inverse limit of an inverse system $\left\{X_{i}, \varphi_{i j}, I\right\}$ of finite discrete spaces. Then $X$ is metrizable if and only if for some countable totally ordered subset $J$ of I (with order type equal to that of $\omega$ ) the space $X$ is the inverse limit of the restricted inverse system $\left\{X_{i}, \varphi_{i j}, J\right\}$.

Proof of Proposition 2.1. By Proposition 2.2, the profinite graph $\Gamma$ is, in the category of profinite graphs, an inverse limit $\lim _{n \geq 1} \Gamma_{n}$ for some inverse sequence of finite graphs $\Gamma_{n}$. By the construction of $\bar{\Omega}_{\Gamma} \mathrm{W}$ given in [1, the semigroupoid $\bar{\Omega}_{\Gamma} \mathrm{W}$ embeds as a closed subsemigroupoid of $\lim _{n>1} \bar{\Omega}_{\Gamma_{n}} \mathrm{~W}$. Since $\Gamma_{n}$ is finite, the profinite semigroupoid $\bar{\Omega}_{\Gamma_{n}} \mathrm{~W}$ is metrizable. As the inverse limit of a sequence of metrizable spaces is metrizable (cf. [9, Theorem 22.3]), it follows that $\lim _{n \geq 1} \bar{\Omega}_{\Gamma_{n}} \mathrm{~W}$ and $\bar{\Omega}_{\Gamma} \mathrm{W}$ are metrizable.

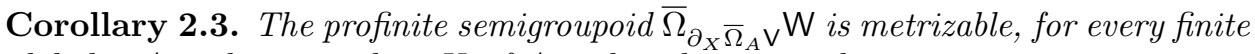
alphabet $A$ and every subset $X$ of $A$ with at least two elements.

Proof. Since $A$ is finite, $\bar{\Omega}_{A} \vee$ is metrizable, and therefore so is $\partial_{X} \bar{\Omega}_{A} \mathrm{~V}$.

The relatively free profinite semigroupoids which intervene in the main results in [2] are of the form $\bar{\Omega}_{\partial_{X} \bar{\Omega}_{A} \vee} \mathrm{W}$. We remark that in (the applications of) Theorem 2.5 of [2, the graph $\partial_{X} \Omega_{A} \vee$ is identified with the graph $\partial_{X}\left(A^{+}\right)$and the sub-

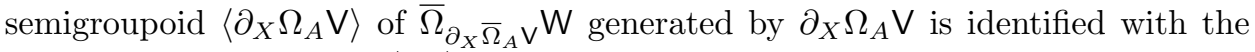
free semigroupoid $\left(\partial_{X}\left(A^{+}\right)\right)^{+}$. These identifications hold in many cases, namely when $\mathrm{V}$ and $\mathrm{W}$ contain all finite nilpotent semigroups: we then have $\Omega_{A} \mathrm{~V} \cong A^{+}$ and $\Omega_{\Gamma} \mathrm{W} \cong \Gamma^{+}$(cf. [1, Theorem 3.16].) But, for example, denoting by Ab the pseudovariety of finite Abelian groups, we know that $\Omega_{A} \mathrm{Ab}$ is not isomorphic to $A^{+}$. To fix this problem it suffices to replace $\partial_{X}\left(A^{+}\right)$by $\partial_{X} \Omega_{A} \mathrm{~V}$ and $\left(\partial_{X}\left(A^{+}\right)\right)^{+}$by $\left\langle\partial_{X} \Omega_{A} \mathrm{~V}\right\rangle$ when appropriate in [2].

Another problem in 22 that needs to be fixed stems from the fact that in the

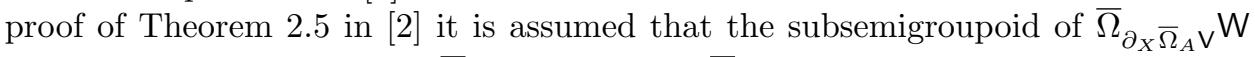
generated by $\partial_{X} \Omega_{A} \vee \subseteq \partial_{X} \bar{\Omega}_{A} \mathrm{~V}$ is dense in $\bar{\Omega}_{\partial_{X} \bar{\Omega}_{A} \mathrm{~V}} \mathrm{~W}$. As already mentioned, after the publication of [2], examples of profinite graphs $\Gamma$ such that $\Omega_{\Gamma} \mathrm{W}$ is not dense in $\bar{\Omega}_{\Gamma} \mathrm{W}$ were given in [1]. Therefore, one needs to verify if the denseness assumption made in the proof of Theorem 2.5 in 2 really holds. In the next proposition, we show that it does hold when $\mathrm{V}$ is a pseudovariety of semigroups such that $\mathrm{SI} \subseteq \mathrm{V} \subseteq \mathrm{CR}$, where $\mathrm{CR}$ denotes the pseudovariety of completely regular semigroups, and such that $\bar{\Omega}_{A} \vee$ has $0, \overline{0}, 1, \overline{1}$ functions for every finite set $A$. 
This suffices to guarantee the main results in Sections 3 to 6 of [2], all about pseudovarieties satisfying these conditions.

Theorem 2.4. Let $\mathrm{V}$ be a pseudovariety of semigroups such that $\mathrm{SI} \subseteq \mathrm{V} \subseteq \mathrm{CR}$ and let $\mathrm{W}$ be any pseudovariety of semigroupoids containing some nontrivial semigroup. Let $A$ be a finite alphabet, and let $X$ be a subset of $A$ with at least two elements. Suppose that $\bar{\Omega}_{A} \vee$ has $0, \overline{0}, 1, \overline{1}$ functions. Then the subsemigroupoid $\left\langle\partial_{X} \Omega_{A} \mathrm{~V}\right\rangle$ of $\bar{\Omega}_{\partial_{X} \bar{\Omega}_{A} \vee} \mathrm{W}$ is dense. In particular, $\Omega_{\partial_{X} \bar{\Omega}_{A} \vee} \mathrm{W}$ is dense in $\bar{\Omega}_{\partial_{X} \bar{\Omega}_{A} \vee} \mathrm{W}$.

Proof. To avoid confusion between the multiplication in $\bar{\Omega}_{A} \mathrm{~V}$ and the edge multi-

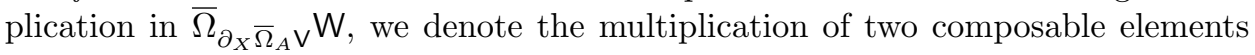
$e, f$ of $\bar{\Omega}_{\partial_{X} \bar{\Omega}_{A} \vee} \mathrm{W}$ by $e \circ f$.

Note that $\partial_{X} \bar{\Omega}_{A} \mathrm{~V}=\overline{\partial_{X} \Omega_{A} \mathrm{~V}}$, so that, in particular, $\overline{\left\langle\partial_{X} \Omega_{A} \mathrm{~V}\right\rangle}$ contains $\partial_{X} \bar{\Omega}_{A} \mathrm{~V}$. Therefore, since $\bar{\Omega}_{\partial_{X} \bar{\Omega}_{A} \vee} \mathrm{W}$ is the unique closed subsemigroupoid of $\bar{\Omega}_{\partial_{X} \bar{\Omega}_{A} \vee} \mathrm{W}$ containing $\partial_{X} \bar{\Omega}_{A} \mathrm{~V}$, to show that $\left\langle\partial_{X} \Omega_{A} \mathrm{~V}\right\rangle$ is dense in $\bar{\Omega}_{\partial_{X} \bar{\Omega}_{A} \vee} \mathrm{W}$ it suffices to show that $\overline{\left\langle\partial_{X} \Omega_{A} \mathrm{~V}\right\rangle}$ is a subsemigroupoid of $\bar{\Omega}_{\partial_{X} \bar{\Omega}_{A} \vee} \mathrm{W}$.

Thanks to Corollary 2.3. what we want to show translates into proving that if two edges $e$ and $f$ of $\bar{\Omega}_{\partial_{X} \bar{\Omega}_{A}} \vee \mathrm{W}$ are limits of sequences $\left(e_{n}\right)_{n}$ and $\left(f_{n}\right)_{n}$ of elements of $\left\langle\partial_{X} \Omega_{A} \mathrm{~V}\right\rangle$ such that $e \circ f$ is defined, then $e \circ f$ is also the limit of a sequence of elements of $\left\langle\partial_{X} \Omega_{A} \mathrm{~V}\right\rangle$. This property is established if one shows that it is possible to replace each $f_{n}$ by another element $\tilde{f}_{n}$ of $\left\langle\partial_{X} \Omega_{A} \mathrm{~V}\right\rangle$ such that $e_{n} \circ \tilde{f}_{n}$ is defined and $\left(\tilde{f}_{n}\right)_{n}$ converges to $f$. The claim follows by continuity of edge multiplication

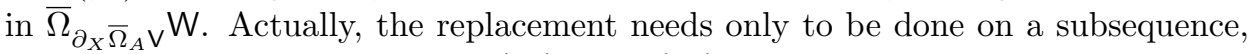
so we may take subsequences of $\left(e_{n}\right)_{n}$ and $\left(f_{n}\right)_{n}$ as convenient.

The only relevant part of $e$ is its end vertex $u$. We denote by $u_{n}$ the end vertex of $e_{n}$ and by $u_{n}^{\prime}$ the beginning vertex of $f_{n}$. We then have $\lim u_{n}=u=\lim u_{n}^{\prime}$. Since the content function is continuous, by taking subsequences we may assume that $c\left(u_{n}\right)=c(u)=c\left(u_{n}^{\prime}\right)$ for every $n$. Since $f_{n} \in\left\langle\partial_{X} \Omega_{A} \mathrm{~V}\right\rangle$, there is a factorization $f_{n}=s_{n} \circ t_{n}$ such that $s_{n} \in \partial_{X} \Omega_{A} \mathrm{~V}$ and $t_{n} \in\left\langle\partial_{X} \Omega_{A} \mathrm{~V}\right\rangle \uplus\left\{I_{n}\right\}$, where $I_{n}$ denotes an adjoined local identity. Again taking subsequences, we may assume that $\left(s_{n}\right)_{n}$ converges to $s$ and $\left(t_{n}\right)_{n}$ converges to $t$ (where $t$ may be an adjoined local identity $I$.) Note that, by continuity of $\circ$, we have $f=s \circ t$. Consider factorizations of the form

$$
s_{n}=0\left(s_{n}\right) \overline{0}\left(s_{n}\right) x_{n}=y_{n} \overline{1}\left(s_{n}\right) 1\left(s_{n}\right) \text {, }
$$

with $x_{n}, y_{n} \in\left(\bar{\Omega}_{A} \mathrm{~V}\right)^{1}$. We have $0\left(s_{n}\right)=\alpha\left(s_{n}\right)=u_{n}^{\prime}$ and $\omega\left(s_{n}\right)=1\left(s_{n}\right)=\alpha\left(t_{n}\right)$. Once again by taking subsequences, we may suppose that $\left(x_{n}\right)_{n}$ and $\left(y_{n}\right)_{n}$ respectively converge to some elements $x$ and $y$ of $\left(\bar{\Omega}_{A} \mathrm{~V}\right)^{1}$. Consider the following element of $\bar{\Omega}_{A} \mathrm{~V}$ :

$$
r_{n}=u_{n} \overline{0}\left(s_{n}\right) x_{n} \cdot s_{n}^{n !} .
$$

Since $c\left(r_{n}\right)=c\left(s_{n}\right)$, we know that $r_{n}$ is an edge of $\partial_{X} \Omega_{A} \mathrm{~V}$ such that $1\left(r_{n}\right)=1\left(s_{n}\right)$, and we also have $0\left(r_{n}\right)=u_{n}$ due to the equality $c\left(u_{n}\right)=c\left(0\left(s_{n}\right)\right)$. Therefore, we may define the element $e_{n} \circ r_{n} \circ t_{n}$ of $\left\langle\partial_{X} \Omega_{A} \mathrm{~V}\right\rangle$. By continuity of $\overline{0}$, we have

$$
\lim u_{n} \overline{0}\left(s_{n}\right) x_{n}=u \overline{0}(s) x=\lim 0\left(s_{n}\right) \overline{0}\left(s_{n}\right) x_{n}=s .
$$

On the other hand, the sequence $\left(s_{n}^{n !}\right)_{n}$ converges to the idempotent $s^{\omega}$. Therefore, we have $\lim r_{n}=s^{\omega+1}=s$, where the last equality holds because $\mathrm{V} \subseteq \mathrm{CR}$. Hence, we obtained a sequence $\left(e_{n} \circ r_{n} \circ t_{n}\right)_{n}$ of elements of $\left\langle\partial_{X} \Omega_{A} \mathrm{~V}\right\rangle$ converging to $e \circ s \circ t=$ $e \circ f$. 


\section{REFERENCES}

[1] J. Almeida and A. Costa. 'Infinite-vertex free profinite semigroupoids and symbolic dynamics'. J. Pure Appl. Algebra 213 (2009), 605-631.

[2] J. Almeida and P. G. Trotter. 'The pseudoidentity problem and reducibility for completely regular semigroups'. Bull. Austral. Math. Soc. 63 (2001), 407-433.

[3] J. Almeida and P. Weil. 'Profinite categories and semidirect products'. J. Pure Appl. Algebra 123 (1998), 1-50.

[4] P. R. Jones. 'Profinite categories, implicit operations and pseudovarieties of categories'. J. Pure Appl. Algebra 109 (1996), 61-95.

[5] J. Rhodes and B. Steinberg. 'Profinite semigroups, varieties, expansions and the structure of relatively free profinite semigroups'. Int. J. Algebra Comput. 11 (2002), 627-672.

[6] J. Rhodes and B. Steinberg. The q-theory of finite semigroups. Springer Monographs in Mathematics (Springer, 2009).

[7] L. Ribes. 'Grupos profinitos y grafos topológicos'. Number 4 in Publicacions de la Secció de Matemàtiques (Universitat Autònoma de Barcelona, 1977), 1-64.

[8] L. Ribes and P. A. Zalesskiı. Profinite Groups. Number 40 in Ergeb. Math. Grenzgebiete 3 (Springer, Berlin, 2000).

[9] S. Willard. General Topology (Addison-Wesley, Reading, Mass., 1970).

CMup, Departamento de Matemática, Faculdade de Ciências, Universidade do Porto, Rua do Campo Alegre 687, 4169-007 Porto, Portugal.

E-mail address: jalmeida@fc.up.pt

CMuC, Department of Mathematics, University of Coimbra, 3001-501 Coimbra, PorTUGAL.

E-mail address: amgc@mat.uc.pt 